\title{
Revisiting Lorentz violation in Hořava gravity
}

\author{
A. Coates, ${ }^{1}$ C. Melby-Thompson, ${ }^{2,3}$ and S. Mukohyama ${ }^{4,5}$ \\ ${ }^{1}$ Theoretical Astrophysics, IAAT, University of Tübingen, Auf der Morgenstelle 10, \\ 72076 Tübingen, Germany \\ ${ }^{2}$ Institut für Theoretische Physik und Astrophysik, Julius-Maximilians-Universität Würzburg, \\ Am Hubland, 97074 Würzburg, Germany \\ ${ }^{3}$ Department of Physics, Fudan University, 220 Handan Road, 200433 Shanghai, China \\ ${ }^{4}$ Center for Gravitational Physics, Yukawa Institute for Theoretical Physics, \\ Kyoto University, 606-8502 Kyoto, Japan \\ ${ }^{5}$ Kavli Institute for the Physics and Mathematics of the Universe (WPI), The University of Tokyo Institutes \\ for Advanced Study, The University of Tokyo, Kashiwa, Chiba 277-8583, Japan
}

(Received 2 June 2019; published 24 September 2019)

\begin{abstract}
In the context of Hořava gravity, the most promising known scenarios to recover Lorentz invariance at low energy are the possibilities that 1) the renormalization group flow of the system leads to emergent infrared Lorentz invariance and 2) that supersymmetry protects infrared Lorentz invariance. A third scenario proposes that a classically Lorentz-invariant matter sector with controlled quantum corrections may simply coexist with Hořava gravity under certain conditions. However, for nonprojectable Hořava gravity in $3+1$ dimensions, it is known that, in the absence of additional structures, this mechanism is spoiled by unexpected power-law divergences. We confirm this same result in the projectable version of the theory by employing the recently found gauge-fixing term that renders the shift and graviton propagators regular. We show that the problem persists for all dimensions $D \geq 3$ and that the degree of fine-tuning in squared sound speeds between a $U(1)$ gauge field and a scalar field increases with $D$. In particular, this difference in the zero external momentum limit is proportional to $\Lambda^{D-1}$ for $D \geq 3$, where $\Lambda$ is the ultraviolet momentum cutoff for loop integrals, while the power-law divergences are absent for $D=1$ and $D=2$. These results suggest that not only the gravity sector but also the matter sector should exhibit a transition to Lifshitz scaling above some scale and that there should not be a large separation between the transition scales in the gravity and matter sectors. We close with a discussion of other more promising scenarios, including emergent Lorentz invariance from supersymmetry/strong dynamics and pointing out challenges where they exist.
\end{abstract}

DOI: $10.1103 /$ PhysRevD.100.064046

\section{INTRODUCTION}

Despite the empirical successes of General Relativity (GR), there are reasons to believe that it is incomplete. The major stumbling block from a theoretical perspective is that quantum GR is not perturbatively renormalizable. One approach to improving its UV properties is to relinquish Lorentz invariance at short distances [1], allowing the inclusion of higher order derivatives that render gravity renormalizable without violating unitarity. Renormalization group (RG) flow is organized around a fixed point with "Lifshitz scaling," which acts anisotropically on space and time, so that higher order spatial derivatives scale

Published by the American Physical Society under the terms of the Creative Commons Attribution 4.0 International license. Further distribution of this work must maintain attribution to the author(s) and the published article's title, journal citation, and DOI. Funded by SCOAP . the same way as second order time derivatives in the ultraviolet $[1,2] .{ }^{1}$ We refer to such theories as Hořava gravity. Hořava gravity comes in two basic flavors: the "projectable" and "nonprojectable" varieties. Both versions remain viable, and the most recent treatments of their observational constraints can be found in Refs. [5] and [6,7], respectively.

Lorentz violations in the matter sector, however, are very tightly constrained. For example, the relative differences among speed limits for different species of matter fields are typically constrained to be smaller than $10^{-23}$ or so. Moreover, thanks to the recent multimessenger observation of a binary neutron star merger, we now know that even the speed of gravitational waves cannot be different from that

\footnotetext{
${ }^{1}$ The Lifshitz scaling is also the origin of interesting cosmological implications of the theory, such as a novel mechanism for generating scale-invariant perturbations [3] and a solution to the flatness problem [4]. See Ref. [5] for a review of cosmology based on Hořava gravity.
} 
of photons by more than $10^{-15}$ or so. Therefore, naturalness demands a mechanism either protecting infrared Lorentz symmetry or causing it to emerge $[8,9]$ in the infrared. If Hořava gravity or something similar is the correct description of gravity in the nature, those constraints require the RG flow of Lorentz-violating couplings to vanish rapidly in the IR [10]. This may be the case if supersymmetry protects infrared Lorentz invariance [11] and/or the system passes through a strongly coupled region which speeds up the otherwise logarithmic RG running of Lorentz-violating couplings toward zero $[12,13]$. We briefly discuss these possibilities in Sec. IV.

There is yet another possibility that, until recently, seemed quite promising: the mechanism proposed in Ref. [14]. The basic statement of this mechanism is that if one assumes that matter is relativistic at tree level then Planck suppression of interactions between gravity and matter can lead to a suppression of Lorentz-violating corrections by the ratio

$$
\frac{\delta c^{2}}{c^{2}} \sim\left(\frac{M_{*}}{M_{p}}\right)^{2} \sim 10^{-18}\left(\frac{M_{*}}{10^{10} \mathrm{GeV}}\right)^{2},
$$

where $M_{*}$ is the scale suppressing the higher order spatial derivatives in the gravity sector. However, it was already shown in Ref. [14] that the vector sector of the nonprojectable version of Hořava gravity does not benefit from this mechanism. Instead, one finds divergences of the form

$$
\frac{\delta c^{2}}{c^{2}} \sim\left(\frac{\Lambda}{M_{p}}\right)^{2}
$$

where $\Lambda$ is the ultraviolet momentum cutoff. There, where the temporal gauge was used, this was attributed to the lack of Lifshitz scaling in the vector sector. ${ }^{2}$

The authors of Ref. [14] proposed eliminating the powerlaw divergences by introducing mixed derivatives into the Hořava action, in the form of terms quadratic in $\nabla_{k} K_{i j}\left(K_{i j}\right.$ is the extrinsic curvature of the spatial slices); see also Refs. [15,16]. In the nonprojectable model, however, radiative corrections in the presence of mixed derivatives are expected to give rise to terms quadratic in time derivatives of the lapse $\mathcal{N}$, leading to a new scalar degree of freedom (d.o.f.) that is unstable in the IR [17]. The viability of the mechanism, as presented in Ref. [14] for the nonprojectable theory, therefore needs a more in-depth analysis. We shall briefly discuss this point in Sec. IVA.

The present paper has two main goals. The first is to investigate the same mechanism in the projectable version of Hořava gravity in $D+1$ dimensions. The projectable theory has technical advantages over the nonprojectable theory, not the least of which is that it is known to be

\footnotetext{
${ }^{2}$ The authors state, however, that they verified their computations in a generalization of the $R_{\xi}$ gauge.
}

renormalizable [18], while renormalizability of the nonprojectable theory remains an open question. A technical but crucial difference between the two theories is that only in the projectable case does the gravity sector possess propagators that are regular, explicitly respect the Lifshitz scaling in the ultraviolet, and have no instantaneous modes, ${ }^{3}$ provided we adopt the gauge fixing of [18]. It is therefore beneficial to revisit Lorentz violation in the matter sector due to quantum corrections in the context of the projectable version of the theory. We shall confirm that the divergence structure is robust and that the problem persists in $D+1$ dimensions for any $D \geq 3$.

Our second goal is to discuss possible resolutions of these problems. These fall into three classes: mixed derivative terms in the projectable Hořava gravity, supersymmetry, and strong dynamics. The mixed derivative terms proposed in Ref. [14] for the nonprojectable model do not introduce a new scalar d.o.f. in the projectable theory because there the offending term does not exist. One is instead faced with infrared instabilities, imposing strong conditions on the value of $\lambda$. The viability of the mechanism then reduces to the RG properties of $\lambda$, together with the existence of an analogue of the Vainshtein mechanism rendering the dynamics at $\lambda$ near 1 regular. A second option, first investigated in Ref. [11], is the use of supersymmetry to control Lorentz violation in the infrared, and we comment on the possibility of combining the mechanisms of Refs. $[11,14,19]$ to suppress the loop contributions of the shift variable. Finally, we briefly discuss the strong coupling mechanism studied in Refs. [12,13].

The rest of the paper is organized as follows. Section II introduces our models, consisting of Hořava gravity coupled to a free scalar and a $U(1)$ gauge field, respectively; gives their propagators in the gauge fixing of Ref. [18], and lists the integrals that appear when calculating divergent contributions to the one-loop effective action. Section III computes the one-loop corrections to the scalar and gauge limiting speeds induced by the gravitational sector. Possible solutions to the naturalness problem for Lorentz symmetry are discussed in Sec. IV, and Sec. V summarizes our conclusions and discusses interesting directions for future work.

\section{SETUP AND NOTATION}

Our treatment of Hořava gravity follows the setup of Ref. [18] closely. We shall only take the extrinsic curvature terms and the terms which are of order $2 D$ in spatial derivatives, as we are interested only in the UV contributions of gravitational degrees to loop integrals. We shall use the gauge-fixing prescription of Ref. [18], for which all

\footnotetext{
${ }^{3}$ In the nonprojectable theory, there exist modes of which the momentum space correlators diverge with respect to spatial momentum even at nonzero frequency $\omega$, which leads to instantaneous propagation.
} 
propagators are regular. On the other hand, we need not worry about introducing Faddeev-Popov ghost fields, since at one loop they do not contribute to the matter sector effective action. As we consider the projectable version of the theory, we are also free to set the lapse, $\mathcal{N}$, to 1 throughout the paper. We shall work in the Euclidean signature, so $\mathcal{L}_{\mathcal{V}}$ below has a minus sign relative to the real time action. We then add a matter sector that (before Wick rotation) is Lorentz invariant at the classical level and investigate Lorentz-violating quantum corrections in the zero external momentum limit.

\section{A. Gravity action}

Hořava gravity starts with a fixed foliation of spacetime by constant time slices. In terms of a coordinate system $\left(t, x^{i}\right)$ respecting the foliation, it is built from the ADM fields $\mathcal{N}, \mathcal{N}_{i}$, and $g_{i j}$. We work solely with the so-called projectable theory, where $\mathcal{N}=\mathcal{N}(t)$ is constant on spatial slices. Its action is

$S_{H L}=\frac{1}{2 \kappa^{2}} \int d t d^{D} x \mathcal{N} \sqrt{g}\left[K_{i j} K^{i j}-\lambda K^{2}+\mathcal{L}_{V}+\mathcal{L}_{\text {g.f. }}\right]$,

where $K_{i j}=\frac{1}{2 \mathcal{N}}\left(\dot{g}_{i j}-\nabla_{i} \mathcal{N}_{j}-\nabla_{j} \mathcal{N}_{i}\right)$ and $\mathcal{L}_{V}$ is built from the Riemann curvature tensor of the spatial slice using terms containing $2 D$ spatial derivatives. The coupling constants $\lambda$ and $\kappa^{2}$ are dimensionless under the UV scaling.

To compute the leading divergent contributions to the matter effective action, it is enough to set the cosmological constant to zero and expand around a flat background,

$$
\mathcal{N}=1, \quad \mathcal{N}_{i}=N_{i}, \quad g_{i j}=\delta_{i j}+h_{i j},
$$

where $N_{i}$ and $h_{i j}$ are small perturbations. The gauge-fixing term of Ref. [18] has two free parameters, $\sigma$ and $\xi$, and takes the form

$$
\mathcal{L}_{\text {g.f. }}=\sigma F^{i} \mathcal{O}_{i j} F^{j},
$$

where

$$
\begin{gathered}
\mathcal{O}_{i j}=\Delta^{-D+2}\left[\delta_{i j} \Delta+\xi \partial_{i} \partial_{j}\right]^{-1}, \\
F^{i}=\dot{N}^{i}+\frac{1}{2 \sigma} \mathcal{O}_{i j}^{-1} \partial_{k} h_{j k}-\frac{\lambda}{2 \sigma} \mathcal{O}_{i j}^{-1} \partial_{j} h .
\end{gathered}
$$

This specific choice for the gauge-fixing term decouples the shift and metric perturbations at quadratic order and renders all propagators regular. That this gauge-fixing term takes a universal form depending in a simple way on dimension can be traced to the fact that $N_{i}$ only appears in the kinetic term, which is independent of dimension and the choice of potential in the projectable theory.

\section{B. Matter action}

Following Ref. [14], we take the matter sector to be Lorentz invariant at the Hořava-Lifshitz (HL) scale and evaluate the leading Lorentz-violating quantum corrections in the limit of small external momentum. We will consider two types of matter: a massless scalar and a $U(1)$ gauge field, both taken to be Lorentz invariant in the UV. After Wick rotation, the scalar action takes the form

$$
S_{\phi}=\frac{1}{2} \int d^{D+1} x\left[\left(\partial_{t} \phi-N^{i} \partial_{i} \phi\right)^{2}+\beta^{2} \delta^{i j} \partial_{i} \phi \partial_{j} \phi\right],
$$

plus terms $O\left(h_{i j}\right)$, while the gauge field has the action

$$
S_{A}=\int d^{D+1} x\left[\frac{1}{2}\left(E_{i}-N^{k} F_{k i}\right)^{2}+\frac{\beta^{2}}{4} F_{i j} F_{i j}\right],
$$

again with additional terms $O\left(h_{i j}\right)$ and with the definitions,

$$
E_{i}=D^{E}{ }_{i}^{\mu} A_{\mu}, \quad F_{i j}=D^{F}{ }_{i j}^{\mu} A_{\mu},
$$

where $D^{E}{ }_{i}^{\mu}=\partial_{0} \delta_{i}^{\mu}-\partial_{i} \delta_{0}^{\mu}$ and $D^{F}{ }_{i j}^{\mu}=\partial_{i} \delta_{j}^{\mu}-\partial_{j} \delta_{i}^{\mu}$. Here, we have suppressed the couplings to $h_{i j}$ because, as we argue in the following sections, $h$ loops contribute only logarithmic divergences. The parameter $\beta(>0)$ is the bare propagation speed of $\phi$ and $A_{\mu}$, which we include for bookkeeping purposes.

\section{Propagators}

Because of the particular form of the gauge-fixing term, the shift propagator in Fourier space takes a simple form valid in any dimension,

$$
\begin{aligned}
\tilde{G}_{N}^{i j}\left(P_{\mu}\right) & =a_{1}\left(\delta_{i j}-\hat{p}_{i} \hat{p}_{j}\right) \mathcal{P}_{1}\left(P_{\mu}\right)+a_{2} \hat{p}_{i} \hat{p}_{j} \mathcal{P}_{2}\left(P_{\mu}\right), \\
a_{1} & =\frac{\kappa^{2}}{\sigma}, \quad a_{2}=\frac{\kappa^{2}(1+\xi)}{\sigma},
\end{aligned}
$$

where $P_{\mu}=\left(\omega, p_{i}\right)$ is the 4-momentum, $\hat{p}_{i}=p_{i} / p$, $p=\sqrt{\delta^{i j} p_{i} p_{j}}$, and

$$
\begin{aligned}
\mathcal{P}_{1,2}\left(P_{\mu}\right) & =\frac{p^{2(D-1)}}{\omega^{2}+\alpha_{1,2}^{2} p^{2 D}}, \\
\alpha_{1}^{2} & =\frac{1}{2 \sigma}, \quad \alpha_{2}^{2}=\frac{(1-\lambda)(1+\xi)}{\sigma} .
\end{aligned}
$$

For the graviton $h_{i j}$, any potential $\mathcal{L}_{\mathcal{V}}$ satisfying physically reasonable inequalities leads to a well-behaved Lifshitz dispersion relation, $\omega^{2} \propto k^{2 D}$, for all modes. This leads to a Fourier-space propagator of the form

$$
G_{i j k \ell}^{h}\left(P_{\mu}\right)=\sum_{n} M_{i j k \ell}^{(n)} \mathcal{P}_{n}^{\prime}\left(P_{\mu}\right),
$$


where $M_{i j k l}^{(n)}$ runs over a set of tensor structures (projecting onto transverse traceless, divergenceless vector, and two scalar modes), and the $\mathcal{P}_{n}^{\prime}$ are all of the form

$$
\mathcal{P}_{n}^{\prime}\left(P_{\mu}\right)=\frac{a_{n}^{\prime}}{\omega^{2}+\alpha_{n}^{\prime 2} p^{2 D}} .
$$

The gauge-fixing term is such that the mixed propagator $\left\langle N^{i} h_{j k}\right\rangle$ vanishes.

For a scalar $\phi$ with the action (8), the Fourier space propagator is

$$
\tilde{G}_{\phi}\left(P_{\mu}\right)=\frac{1}{\omega^{2}+\beta^{2} p^{2}},
$$

while a vector field $A_{\mu}$ in Feynman gauge with the action (9) has Fourier-space propagator

$$
\tilde{G}_{A \mu \nu}\left(P_{\rho}\right)=\frac{\eta_{\mu \nu}^{\mathrm{E}}}{\omega^{2}+\beta^{2} p^{2}},
$$

where $\eta_{00}^{\mathrm{E}}=\beta^{2}, \eta_{0 i}^{\mathrm{E}}=\eta_{i 0}^{\mathrm{E}}=0$ and $\eta_{i j}^{\mathrm{E}}=\delta_{i j}$.

\section{Loop integrals}

The leading divergence of the one-loop effective action for $\phi$ and $A_{\mu}$ will be derived in the next section. They can be expressed in terms of the following loop integrals:

$$
\begin{aligned}
\mathcal{J}_{1}^{(I)} & \equiv a_{I} \int \frac{d^{D+1} P}{(2 \pi)^{D+1}} \mathcal{P}_{I}\left(P_{\mu}\right), \\
\mathcal{J}_{2}^{(I)} & \equiv a_{I} \int \frac{d^{D+1} P}{(2 \pi)^{D+1}} \omega^{2} \tilde{G}_{\phi}\left(P_{\mu}\right) \mathcal{P}_{I}\left(P_{\mu}\right), \\
\mathcal{J}_{3}^{(I)} & \equiv a_{I} \int \frac{d^{D+1} P}{(2 \pi)^{D+1}} p^{2} \tilde{G}_{\phi}\left(P_{\mu}\right) \mathcal{P}_{I}\left(P_{\mu}\right), \\
\mathcal{J}_{4}^{(n)} & \equiv \int \frac{d^{D+1} P}{(2 \pi)^{D+1}} \mathcal{P}_{n}^{\prime}\left(P_{\mu}\right), \\
\mathcal{J}_{5}^{(n)} & \equiv \int \frac{d^{D+1} P}{(2 \pi)^{D+1}} \omega^{2} \tilde{G}_{\phi}\left(P_{\mu}\right) \mathcal{P}_{n}^{\prime}\left(P_{\mu}\right), \\
\mathcal{J}_{6}^{(n)} & \equiv \int \frac{d^{D+1} P}{(2 \pi)^{D+1}} p^{2} \tilde{G}_{\phi}\left(P_{\mu}\right) \mathcal{P}_{n}^{\prime}\left(P_{\mu}\right), \\
\mathcal{J}_{7} & \equiv \int \frac{d^{D+1} P}{(2 \pi)^{D+1}} \tilde{G}_{\phi}\left(P_{\mu}\right) .
\end{aligned}
$$

These integrals are regularized and evaluated in Appendix.

\section{ONE-LOOP CORRECTIONS IN $D$ SPATIAL DIMENSIONS}

Our goal is to compute the power-law divergent contributions to the shift in the limiting speeds of the fields $\phi$ and $A_{\mu}$. This is done by computing the one-loop effective action for each field. We will see in Secs. III B and III D that the divergence from $h_{i j}$ is logarithmic, and so the powerlaw divergence comes entirely from the shift $N_{i}$. We therefore begin with loop corrections from the shift field.

\section{A. Shift field loop corrections to the scalar action}

To compute the contribution of shift loops, we may set $h_{i j}=0$ and consider the action

$$
\begin{aligned}
S\left(\phi, N_{i}\right)= & \frac{1}{2} \int d^{D+1} x\left[\left(\partial_{t} \phi-N^{i} \partial_{i} \phi\right)^{2}\right. \\
& \left.+\beta^{2} \delta^{i j} \partial_{i} \phi \partial_{j} \phi+N^{i} \mathcal{K}_{i j} N^{j}\right],
\end{aligned}
$$

where $\mathcal{K}_{i j}$ is the gauge-fixed kinetic term for $N^{i}$. We are interested in the one-loop effective action for $\phi$,

$$
\Gamma_{1}(\phi)=\frac{1}{2} \operatorname{tr}\left(\log S^{(2)}[\phi]-\log S^{(2)}[0]\right),
$$

where $S^{(2)}[\phi]$ is the fluctuation operator, with matrix components

$\left[S^{(2)}[\phi] \cdot f\right](x)=\left.\int d^{D+1} y \frac{\delta^{2} S(\varphi)}{\delta \varphi(x) \delta \varphi(y)}\right|_{N_{i}=0} f(y) ;$

$\varphi$ stands for $\left(\phi, N_{i}\right)$; and $f$ is a test function. Let $G$ denote the propagator matrix, and define the differential operator $U[\phi]$ by

$$
G \cdot S^{(2)}[\phi]=1+G \cdot U[\phi] .
$$

Note that $U[0]=0$. Taylor expanding to second order in $\phi$, we obtain

$$
\Gamma_{1}(\phi)=\frac{1}{2} \operatorname{tr}\left(G \cdot U[\phi]-\frac{1}{2} G \cdot U[\phi] \cdot G \cdot U[\phi]\right) .
$$

The components of $U$ are given by

$$
\begin{aligned}
& \int d^{d+1} x d^{d+1} y f(x) U[\phi]_{\phi(x) \phi(y)} g(y)=0, \\
& \int d^{d+1} x d^{d+1} y f(x) U[\phi]_{\phi(x) N^{i}(y)} v^{i}(y) \\
& =\int d^{d+1} x f(x)\left[\partial_{0}\left(v^{i}(x) \partial_{i} \phi(x)\right)+\partial_{i}\left(v^{i}(x) \partial_{0} \phi(x)\right)\right],
\end{aligned}
$$

$\int d^{d+1} x d^{d+1} y v^{i}(x) U[\phi]_{N^{i}(x) \phi(y)} f(y)$

$$
=\int d^{d+1} x f(x)\left[\partial_{0}\left(v^{i}(x) \partial_{i} \phi(x)\right)+\partial_{i}\left(v^{i}(x) \partial_{0} \phi(x)\right)\right],
$$

$$
\begin{gathered}
\int d^{d+1} x d^{d+1} y v^{i}(x) U[\phi]_{N^{i}(x) N^{j}(y)} u^{j}(y) \\
=\int d^{d+1} x v^{i}(x) u^{j}(x) \partial_{i} \phi(x) \partial_{j} \phi(x),
\end{gathered}
$$




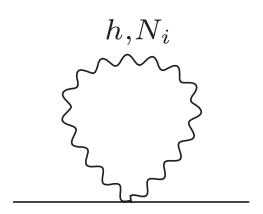

FIG. 1. Single-vertex diagram.

where $f, g, u^{i}$, and $v^{i}$ are test fields. Since the $\phi N_{i}$ Green's function vanishes, the effective action is given to quadratic order in $\phi$ by

$\Gamma_{1}^{(2)}(\phi)=\frac{1}{2} \operatorname{tr}\left(G_{N}^{i j} U_{N^{i} N^{j}}\right)-\frac{1}{2} \operatorname{tr}\left(G_{\phi} U_{\phi N^{i}} G_{N}^{i j} U_{N^{j} \phi}\right)$.

Consider the first trace in (24), corresponding to the diagram in Fig. 1. In the Lifshitz gauge fixing [18], the (momentum space) propagator for $N^{i}$ is a sum of two terms as shown in (11). When $G_{N}^{i j}$ appears inside an integral multiplying a scalar expression, rotational invariance allows us to replace $p_{i} p_{j} \mapsto p^{2} \delta_{i j} / D$. The relevant integrals are defined in Sec. II D and evaluated in Appendix. The final form of the divergent contribution to the effective action from the first term of (24) is therefore

$$
\begin{aligned}
& \int d^{D+1} x G_{N}^{i j}(x, x) \partial_{i} \phi \partial_{j} \phi(x) \\
& =\frac{1}{2} \int d^{D+1} x \zeta_{3} \delta^{i j} \partial_{i} \phi(x) \partial_{j} \phi(x)
\end{aligned}
$$

with

$$
\begin{aligned}
& \zeta_{3}=\frac{\delta_{i j}}{D} G_{N}^{i j}(x, x)=\sum_{I=1}^{2} A_{I} \mathcal{J}_{1}^{(I)}, \\
& A_{1}=1-\frac{1}{D}, \quad A_{2}=\frac{1}{D} .
\end{aligned}
$$

From the second term in (24) (with the diagram in Fig. 2), we obtain a contribution to $\Gamma_{1}(\phi)$ of the form

$$
\begin{aligned}
& -\frac{1}{2} \int d^{D+1} x \int d^{D+1} y\left[\left(-\dot{\phi} \partial_{j}-\partial_{j} \phi \partial_{\tau}\right)^{(x)} G_{\phi}(x, y)\right. \\
& \left.\quad \times\left(\dot{\phi} \partial_{i}+\partial_{i} \phi \partial_{\tau}+2 \partial_{i} \dot{\phi}\right)^{(y)} G_{N}^{i j}(y, x)\right] .
\end{aligned}
$$

From the shift symmetry of $\phi$, we know that all contributions to the Lagrangian must be of the form $D_{1} \phi D_{2} \phi$, where $D_{1}$ and $D_{2}$ are differential operators of order at least 1 . We are interested in the coefficients of $\dot{\phi}^{2}$ and $(\partial \phi)^{2}$. (Note that by time reversal, parity, and rotational symmetry all other contributions to the effective action are less divergent and have more derivatives acting on $\phi$ ). This leaves the leading divergent contribution

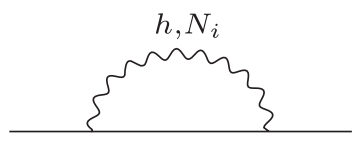

FIG. 2. Two-vertex diagram.

$$
\frac{1}{2} \int d^{D+1} x\left[\zeta_{1}(\dot{\phi})^{2}+\zeta_{2}(\partial \phi)^{2}\right]
$$

For $\zeta_{1}$, we find

$$
\zeta_{1}=\left[\int d^{D+1} y \partial_{j}^{(x)} G_{\phi}(x, y) \partial_{i}^{(y)} G_{N}^{i j}(y, x)\right]_{\mathrm{div}},
$$

which is $-\mathcal{J}_{3}^{(2)}$; note that $\mathcal{P}_{1}$ does not contribute because its prefactor $\left(\delta_{i j}-\hat{p}_{i} \hat{p}_{j}\right)$ inside $G_{N}^{i j}$ is transverse. For $\zeta_{2}$, both $\mathcal{P}_{1}$ and $\mathcal{P}_{2}$ contribute as

$\zeta_{2}=-\left[\frac{\delta_{i j}}{D} \int d^{D+1} y \partial_{\tau}^{(x)} G_{\phi}(x, y) \partial_{\tau}^{(y)} G_{N}^{i j}(y, x)\right]_{\mathrm{div}}$,

which is $-\sum_{I=1}^{2} A_{I} \mathcal{J}_{2}^{(I)}$. We thus find the divergent contribution to the effective action quadratic both in $\phi$ and in derivatives to be

$$
\begin{aligned}
& \Gamma_{1, \text { div }}^{\text {quad }}(\phi) \\
& =\frac{1}{2} \int d^{D+1} x\left[\zeta_{1} \dot{\phi}^{2}+\left(\zeta_{2}+\zeta_{3}\right)(\partial \phi)^{2}\right] \\
& =\frac{1}{2} \int d^{D+1} x\left[-\mathcal{J}_{3}^{(2)} \dot{\phi}^{2}+\sum_{I=1}^{2} A_{I}\left(\mathcal{J}_{1}^{(I)}-\mathcal{J}_{2}^{(I)}\right)(\partial \phi)^{2}\right] .
\end{aligned}
$$

Renormalizing so that $\phi$ has canonical kinetic term $\frac{1}{2} \dot{\phi}^{2}$, we may read off the $N^{i}$-loop contribution to the infrared shift in the limiting speed,

$$
\begin{aligned}
\delta c_{\phi}^{2} & =\zeta_{2}+\zeta_{3}+\beta^{2} \delta Z_{\phi}^{(1)}=\zeta_{2}+\zeta_{3}-\beta^{2} \zeta_{1} \\
& =\sum_{I=1}^{2} A_{I}\left(\mathcal{J}_{1}^{(I)}-\mathcal{J}_{2}^{(I)}\right)+\beta^{2} \mathcal{J}_{3}^{(2)} \\
& =\beta^{2}\left[\left(1-\frac{1}{D}\right) \mathcal{J}_{3}^{(1)}+\left(1+\frac{1}{D}\right) \mathcal{J}_{3}^{(2)}\right],
\end{aligned}
$$

where $\delta Z_{\phi}^{(1)}$ is the $\mathcal{O}(\hbar)$ part of the field renormalization constant. The last equality follows from the first identity of Eq. (A8).

\section{B. Graviton loop corrections to scalar action}

We now show that the one-loop contributions from the graviton $h_{i j}$ to $\delta c_{\phi}^{2}$ do not contain power-law divergences. One-loop gravitational contributions to the matter propagator come in two types, each of which is summed over the contributions of the two graviton modes. [By "graviton," we mean here the modes contributing to the $h_{i j} h_{k \ell}$ correlator (13).] The first is the single-vertex loop, and the second is the two-vertex loop describing the emission and subsequent reabsorption of a virtual graviton (see Figs. 1 and 2). 
In the first case, the loop integral involves only a factor of the graviton propagator, contributing a linear combination of integrals of the form $\mathcal{J}_{4}^{(n)}$, as defined in Sec. II D. These integrals are shown in Appendix to be free from power-law divergences.

The second diagram involves in addition a factor of the scalar propagator. The $h \phi \phi$ vertices are a sum of $\dot{\phi}^{2}$ and $\partial_{i} \phi \partial_{j} \phi$ contributions, and as a result, the diagrams are built from sums of the scalar and graviton propagators, weighted by the square of either the internal scalar energy or momentum. As a result, the leading divergence of these diagrams is given by a linear combination of integrals of the form $\mathcal{J}_{5}^{(n)}$ and $\mathcal{J}_{6}^{(n)}$, also defined in Sec. II D. Again, these contributions are free from power-law divergences, as shown in Appendix. We have thus established that the power-law divergence of the one-loop correction to the infrared limiting speed of the scalar field $\phi$ is given by (32).

\section{Shift field loop corrections to gauge field action}

For shift loop contributions to the gauge field, we once again set $h_{i j}=0$ and consider

$$
\begin{aligned}
S= & \int d^{D+1} x\left[\frac{1}{2}\left(E_{i}-N^{k} F_{k i}\right)^{2}+\frac{\beta^{2}}{4} F_{i j} F_{i j}+N^{i} \mathcal{K}_{i j} N^{j}\right] \\
& +S_{\text {gauge-fixing }},
\end{aligned}
$$

where $E_{i}$ and $F_{i j}$ are defined in (9), $\mathcal{K}_{i j}$ is the gauge-fixed kinetic term for $N^{i}$, and $\beta$ is the same bare propagation speed as before. We adopt the Feynman gauge for $A$, so the propagator is given by (16), although the final result is gauge invariant.

This time, we are interested in the quadratic one-loop effective action for $A$,

$$
\Gamma_{1}^{(2)}(A)=\frac{1}{2} \operatorname{tr}\left(G \cdot U[A]-\frac{1}{2} G \cdot U[A] \cdot G \cdot U[A]\right),
$$

where the $U$ operator has components

$$
\begin{aligned}
& \int d^{d+1} x d^{d+1} y X_{\mu}(x) U[A]_{A_{\mu}(x) A_{\nu}(y)} Y_{\nu}(y)=0 \\
& \int d^{d+1} x d^{d+1} y X_{\mu}(x) U[A]_{A_{\mu}(x) N^{j}(y)} v^{j}(y) \\
& =\int d^{d+1} x X_{\mu}(x)\left[E_{k} D_{j k}^{F \mu} v^{j}+F_{j k} D_{k}^{E \mu} v^{j}+2\left(D^{E}{ }_{k}^{\mu} F_{j k}\right) v^{j}\right] \\
& \int d^{d+1} x d^{d+1} y v^{i}(x) U[A]_{N^{i}(x) A_{\mu}(y)} Y^{\mu}(y) \\
& =-\int d^{d+1} x v^{i}(x)\left[E_{k} D^{F}{ }_{i k}^{\mu} X_{\mu}(x)+F_{i k} D_{k}^{E}{ }_{k}^{\mu} X_{\mu}(x)\right] \\
& \int d^{d+1} x d^{d+1} y v^{i}(x) U[A]_{N^{i}(x) N^{j}(y)} u^{j}(y) \\
& =\int d^{d+1} x v^{i}(x) u^{j}(x) F_{i k} F_{j k} .
\end{aligned}
$$

Here, $D_{i}^{E}{ }_{i}^{\mu}=\partial_{0} \delta_{i}^{\mu}-\partial_{i} \delta_{0}^{\mu}$ and $D^{F}{ }_{i j}^{\mu}=\partial_{i} \delta_{j}^{\mu}-\partial_{j} \delta_{i}^{\mu}$ as in Sec. II B, and $X_{\mu}, Y_{\mu}, u^{i}$, and $v^{i}$ are test fields. We first compute

$$
\begin{aligned}
\frac{1}{2} \operatorname{tr}(G \cdot U[A]) & =\frac{1}{2} \operatorname{tr}_{N}\left(G_{N} \cdot U_{N N}[A]\right) \\
& =\frac{1}{2} \int d^{D+1} x G_{N}^{i j}(x, x)\left(F_{i k} F_{j k}\right)(x) \\
& =\frac{1}{4} \zeta_{3}^{A} \int d^{D+1} x F_{i j} F_{i j},
\end{aligned}
$$

where $\zeta_{3}^{A}=2 \zeta_{3}^{\phi}$. Next, we have the $E_{i}^{2}$ contribution. Pulling this out of the analog of (27), we have

$$
\begin{aligned}
- & \left.\frac{1}{2} \operatorname{tr}\left(G_{N} \cdot U_{N A} \cdot G_{A} \cdot U_{A N}\right)\right|_{E^{2}} \\
= & \frac{1}{2} \int d^{D+1} x \int d^{D+1} y\left[\left(E_{\ell} D_{j \ell}^{F}{ }_{j}^{\mu}\right)^{(x)} G_{\mu \nu}^{A}(x, y)\right. \\
& \left.\times\left(E_{k} D^{F}{ }_{i k}\right)^{(y)} G_{N}^{i j}(y, x)\right] .
\end{aligned}
$$

As before, the dominant divergence is

$$
\frac{1}{2} \int d^{D+1} x E_{k} E_{\ell} Z^{k \ell},
$$

where $Z^{k \ell}=\left(\tilde{Z}^{k \ell}+\tilde{Z}^{\ell k}\right) / 2$ and

$$
\tilde{Z}^{k \ell}=\int d^{D+1} y D_{j \ell}^{F}{ }_{j \ell}^{\mu(x)} G_{\mu \nu}^{A}(x, y) D_{i k}^{F}{ }_{i k}^{\nu(y)} G_{N}^{i j}(y, x) .
$$

By rotational invariance, $Z^{k \ell}=\delta_{k \ell} \zeta_{1}^{A}$, where

$$
\begin{aligned}
\zeta_{1}^{A}= & \frac{1}{D} \delta_{k \ell} Z^{k \ell} \\
= & \frac{1}{D} \int d^{D+1} y \int d^{D+1} y D_{j k}^{F}{ }_{j k}^{(x)} G_{\mu \nu}^{A}(x, y) D^{F}{ }_{i k}^{\nu(y)} G_{N}^{i j}(y, x) \\
= & -\frac{1}{D} \int \frac{d \omega d^{D} p}{(2 \pi)^{D+1}} \frac{\left(p_{j} \eta_{k \nu}^{\mathrm{E}}-p_{k} \eta_{j \nu}^{\mathrm{E}}\right)\left(p_{i} \delta_{k}^{\nu}-p_{k} \delta_{i}^{\nu}\right)}{\omega^{2}+\beta^{2} p^{2}} \\
& \times\left[a_{1}\left(\delta_{i j}-\hat{p}_{i} \hat{p}_{j}\right) \mathcal{P}_{1}+a_{2} \hat{p}_{i} \hat{p}_{j} \mathcal{P}_{2}\right] \\
= & -\left(1-\frac{1}{D}\right) \int \frac{d \omega d^{D} p}{(2 \pi)^{D+1}} \frac{p^{2}}{\omega^{2}+\beta^{2} p^{2}} \\
& \times \delta^{i j}\left[a_{1}\left(\delta_{i j}-\hat{p}_{i} \hat{p}_{j}\right) \mathcal{P}_{1}+a_{2} \hat{p}_{i} \hat{p}_{j} \mathcal{P}_{2}\right] \\
= & -\left(1-\frac{1}{D}\right) \sum_{I=1}^{2} \mathcal{J}_{3}^{(I)}
\end{aligned}
$$

Similarly, the $F^{2}$ term is

$$
\begin{aligned}
& \frac{1}{2} \int d^{D+1} x \int d^{D+1} y\left[\left(F_{j \ell} D^{E}{ }_{\ell}^{\mu}\right)^{(x)} G_{\mu \nu}^{A}(x, y)\right. \\
& \left.\quad \times\left(F_{i k} D_{k}^{E}\right)^{(y)} G_{N}^{i j}(y, x)\right],
\end{aligned}
$$


with leading divergence

$$
\frac{1}{4} \int d^{3} x F_{i k} F_{j \ell} Z^{i k, j \ell}
$$

where $Z^{i k, j \ell}=\left[\left(\tilde{Z}^{i k, j \ell}-\tilde{Z}^{i k, \ell j}-\tilde{Z}^{k i, j \ell}+\tilde{Z}^{k i, \ell j}\right)+\left(\tilde{Z}^{j \ell, i k}-\right.\right.$ $\left.\left.\tilde{Z}^{j \ell, k i}-\tilde{Z}^{\ell j, i k}+\tilde{Z}^{\ell j, k i}\right)\right] / 8$ and $\tilde{Z}^{i k, j \ell}$ is given by

$$
\left.2 \int d^{D+1} y D_{\ell}^{E}{ }_{\ell}^{(x)} G_{\mu \nu}^{A}(x, y) D_{k}^{E \nu(y)} G_{N}^{i j}(y, x)\right|_{\text {div }} .
$$

Using $\eta_{00}^{\mathrm{E}}=\beta^{2}, \eta_{0 i}^{\mathrm{E}}=\eta_{i 0}^{\mathrm{E}}=0$, and $\eta_{i j}^{\mathrm{E}}=\delta_{i j}$, we obtain

$$
\begin{aligned}
\tilde{Z}^{i k, j \ell}= & -2 \int \frac{d \omega d^{D} p}{(2 \pi)^{D+1}} \frac{\left(\omega \delta_{\ell}^{\mu}-\delta_{0}^{\mu} p_{\ell}\right) \eta_{\mu \nu}^{\mathrm{E}}}{\omega^{2}+\beta^{2} p^{2}}\left(\omega \delta_{k}^{\nu}-p_{k} \delta_{0}^{\nu}\right) \\
& \times\left[a_{1}\left(\delta_{i j}-\hat{p}_{i} \hat{p}_{j}\right) \mathcal{P}_{1}+a_{2} \hat{p}_{i} \hat{p}_{j} \mathcal{P}_{2}\right] \\
= & -2 \int \frac{d \omega d^{D} p}{(2 \pi)^{D+1}} \frac{\omega^{2} \delta_{k \ell}+\beta^{2} p_{k} p_{\ell}}{\omega^{2}+\beta^{2} p^{2}} \\
& \times\left[a_{1}\left(\delta_{i j}-\hat{p}_{i} \hat{p}_{j}\right) \mathcal{P}_{1}+a_{2} \hat{p}_{i} \hat{p}_{j} \mathcal{P}_{2}\right]
\end{aligned}
$$

For each tensor structure inside the integral, we can replace

$$
p_{i} p_{j} \rightarrow \frac{1}{D} p^{2} \delta_{i j}, \quad p_{i} p_{j} p_{k} p_{\ell} \rightarrow 0
$$

where the latter comes from contracting with $F_{i k}$. Taking into account index symmetries, the final result can be written as $Z^{i k, j \ell}=\zeta_{2}^{A}\left(\delta_{i j} \delta_{k \ell}-\delta_{i \ell} \delta_{k j}\right) / 2$. The contribution to $\zeta_{2}^{A}$ from the $\mathcal{P}_{1}$ term is

$$
\begin{gathered}
-2 \int \frac{d \omega d^{D} p}{(2 \pi)^{D+1}} \frac{p^{2(D-1)}\left(\omega^{2}\left(1-\frac{1}{D}\right)+\beta^{2} p^{2}\left(\frac{1}{D}\right)\right)}{\left(\omega^{2}+\alpha_{1}^{2} p^{2 D}\right)\left(\omega^{2}+\beta^{2} p^{2}\right)} \\
=-2\left[\left(1-\frac{1}{D}\right) \mathcal{J}_{1}^{(1)}-\left(1-\frac{2}{D}\right) \beta^{2} \mathcal{J}_{3}^{(1)}\right],
\end{gathered}
$$

while the contribution from the $\mathcal{P}_{2}$ term is

$$
-\frac{2}{D} \int \frac{d \omega d^{D} p}{(2 \pi)^{D+1}} \frac{\omega^{2} p^{2(D-1)}}{\left(\omega^{2}+\alpha_{1}^{2} p^{2 D}\right)\left(\omega^{2}+\beta^{2} p^{2}\right)},
$$

which is simply $-\frac{2}{D} \mathcal{J}_{2}^{(2)}$. This gives the net result

$\zeta_{2}^{A}=-2\left[\left(1-\frac{1}{D}\right) \mathcal{J}_{1}^{(1)}-\beta^{2}\left(1-\frac{2}{D}\right) \mathcal{J}_{3}^{(1)}+\frac{1}{D} \mathcal{J}_{2}^{(2)}\right]$.

Combining these results and the contribution from field renormalization, one obtains

$$
\begin{aligned}
\delta c_{A}^{2} & =\left(\zeta_{2}^{A}+\zeta_{3}^{A}\right)-\beta^{2} \zeta_{1}^{A} \\
& =\beta^{2}\left[\left(3-\frac{5}{D}\right) \mathcal{J}_{3}^{(1)}+\left(1+\frac{1}{D}\right) \mathcal{J}_{3}^{(2)}\right] .
\end{aligned}
$$

Once again, we have used the first identity of (A8).

\section{Graviton loop corrections to gauge field action}

The argument given in Sec. III B that the graviton modes do not contribute power-law divergences to $\delta c_{\phi}^{2}$ carries over to the gauge field without modification. Therefore, the power-law divergence of the one-loop correction to the infrared limiting speed of the gauge field $A_{\mu}$ is given by (49).

\section{E. Lorentz-violating quantum corrections}

To summarize, we find that the shift loop contributions (32) and (49) generate power-law divergences in the difference in squared sound speeds between a $U(1)$ gauge field and a scalar field, of the form

$$
\delta c_{A}^{2}-\delta c_{\phi}^{2}=\frac{2(D-2)}{D} \mathcal{J}_{3}^{(1)}
$$

This vanishes only for $D=1$, where $\mathcal{J}_{3}^{(1)}$ vanishes because of the absence of the first term $\left[\propto\left(p^{2} \delta_{i j}-p_{i} p_{j}\right)=0\right]$ in (11), and for $D=2$. As shown in Appendix, the integral $\mathcal{J}_{3}^{(1)}$ diverges as approximately $\Lambda^{D-1}$, where $\Lambda$ is the ultraviolet momentum cutoff. Therefore, we have a naturalness problem for all $D \geq 3$. For $D=3$ (where $M_{p}^{2}=1 / \kappa^{2}$ ), this reduces to

$$
\delta c_{A}^{2}-\delta c_{\phi}^{2}=\frac{1}{4 \pi^{2}}\left(\frac{\Lambda}{M_{p}}\right)^{2} .
$$

\section{POSSIBLE SOLUTIONS}

In this section, we discuss some possible resolutions coming from modification of the gravity theory. Since we have shown that the naturalness problem for Lorentzviolating couplings only becomes worse for $D>3$, in what follows, we shall focus on $D=3$.

\section{A. Mixed derivatives}

The first proposed solution to this naturalness problem, presented in the original paper, was to introduce mixed derivative terms [14]. In nonprojectable Hořava gravity, the $T$-invariant mixed derivative terms of lowest dimension are quadratic in the objects

$$
\nabla_{i} K_{j k}, \quad \mathcal{A}_{i} \equiv \frac{1}{2 \mathcal{N}}\left(\dot{a}_{i}-\mathcal{N}^{j} \nabla_{j} a_{i}-a_{j} \nabla_{i} \mathcal{N}^{j}\right),
$$


where $a_{i} \equiv \partial_{i} \ln \mathcal{N}$. Unfortunately, the term quadratic in the latter object,

$$
\frac{M_{p}^{2} \sigma_{1}}{M^{2}} g^{i j} \mathcal{A}_{i} \mathcal{A}_{j}
$$

generates a new scalar d.o.f. possessing an IR instability [17]. If we preserve the stability criteria of the theory without mixed derivatives while avoiding ghosts, the instability comes from the relative sign of the $\mathcal{A}^{2}$ term and the term proportional to $a^{2}$.

In the projectable version of Horava gravity, on the other hand, the lapse function $\mathcal{N}$ is constant in space, and thus the offending term (53) does not arise. However, the projectable version of Hořava gravity without mixed derivative terms is also known to have an infrared gradient instability. Since mixed derivative terms are irrelevant in the infrared, their presence does not affect the nature of infrared instabilities. For the theory to be phenomenologically viable, the timescale of the infrared instability must be longer than either the Hubble timescale or the standard Jeans timescale. In $3+1$ dimensions, this leads to the following nontrivial condition on the RG flow of $\lambda$ [5],

$$
0<\frac{\lambda-1}{3 \lambda-1}<\max \left[\frac{H^{2}}{k^{2}},|\Phi|\right]
$$

for,

$$
H<k<\min \left[M, \frac{1}{0.01 \mathrm{~mm}}\right],
$$

where $k$ is the momentum scale of interest, $H$ is the Hubble expansion rate of the background, $\Phi$ is the Newton potential, and $M$ is the Lifshitz scale. This in particular implies that $\lambda$ should run toward 1 sufficiently rapidly in the infrared. In the limit $\lambda \rightarrow 1$, the perturbative expansion breaks down, and a fully nonlinear analysis is needed. In general, such nonlinear analyses are technically difficult. Fortunately, in some simple cases, such as stationary spherically symmetric configurations [5] and nonlinear superhorizon perturbations [20,21], fully nonlinear analyses have been performed, and it has been shown that general relativity (plus "dark matter as integration constant" $[22,23])$ is recovered in the limit $\lambda \rightarrow 1$. It is therefore worthwhile to investigate the naturalness problem in the projectable theory with mixed derivative terms.

A similar constraint can be placed in the nonprojectable theory, giving

$$
0<M^{2} \frac{\alpha}{\sigma_{1}}<\frac{1}{4} \max \left[H^{2}, k^{2}|\Phi|\right]
$$

for

$$
H<k<\min \left[M, \frac{1}{0.01 \mathrm{~mm}}\right]
$$

where $\sigma_{1}$ was defined in (53) and $\alpha$ is the coefficient of $a_{i} a^{i}$ [24]. Treating this is as a constraint on the IR behavior of $\alpha$, one obtains

$$
\alpha \lesssim 10^{-104}\left(\frac{10^{10} \mathrm{GeV}}{M}\right)^{2}\left(\frac{\sigma_{1}}{1}\right) \max \left[\frac{H^{2}}{H_{0}^{2}}, \frac{k^{2}|\Phi|}{H_{0}^{2}}\right],
$$

where $H_{0} \sim 10^{-42} \mathrm{GeV}$ is the present value of $H$.

If one likes to avoid technical complications due to nonlinearity of the scalar graviton in establishing the lowenergy recovery of general relativity, then the $U(1)$ extension [25] may provide a way out since there is no scalar graviton in the $U(1)$ extension with projectability. ${ }^{4}$ In this case, matter can couple universally to a gauge-invariant variable constructed from the gauge field and the so-called Newtonian prepotential at low energy, e.g., after integrating out heavy fields. ${ }^{5}$ It has been shown that all the solar system tests carried out so far are satisfied in a large region of the parameter space [26]. It is therefore worthwhile to investigate the naturalness problem in the context of the projectable $U(1)$ extension, with or without mixed derivative terms, in future work.

\section{B. Supersymmetry}

One promising mechanism to protect infrared Lorentz invariance is to impose supersymmetry [11]. While the supersymmetry (SUSY) algebra is usually treated as an extension of Poincaré symmetry, supersymmetry does not imply Lorentz symmetry. It was shown in Refs. $[11,28]$ that, in a four-dimensional theory with $\mathcal{N}=1$ SUSY, if all fields are charged under a gauge symmetry and there is at most one $U(1)$ gauge field, then Lorentz-violating (LV) operators have dimension greater than or equal to 5. If $C P T$ is imposed, ${ }^{6}$ the lower bound on LV operator dimensions becomes 6 . We assume $C P T$ in what follows since $C P T$ violation is rather strongly constrained, e.g., by observation of polarized gamma rays from distant gamma-ray bursts (see Ref. [29] and references therein).

If SUSY is a UV symmetry of nature, then it must be broken at some point, generating LV effects below the breaking scale. These effects must be suppressed in order to maintain IR Lorentz invariance. When SUSY is softly broken at scale $m_{\text {soft }}$, dimension- 4 operators are generated but are suppressed by the ratio $m_{\mathrm{soft}}^{2} / \Lambda_{\mathrm{LV}}^{2}$, where $\Lambda_{\mathrm{LV}}$ is the LV scale [14,19]. This protects IR Lorentz symmetry,

\footnotetext{
${ }^{4}$ On the other hand, there is a scalar graviton in the nonprojectable version of the $U(1)$ extension [26,27].

${ }^{5}$ See Appendix C of Ref. [26] for a simple example.

${ }^{6}$ The $C P T$ theorem assumes Lorentz invariance. Therefore, in a Lorentz-violating theory, $C P T$ invariance is a nontrivial condition we are free to impose.
} 
provided that $m_{\text {soft }} \ll \Lambda_{\mathrm{LV}}$ and that $\Lambda_{\mathrm{LV}}$ is sufficiently large that the dimension- 6 operators satisfy observational constraints. Taking energy dependence of gamma-ray burst light curves [30], the suppression scale of the dimension-6 operator, which is expected to be of order $\Lambda_{\mathrm{LV}}$ unless fine tuned, is constrained as $M_{\mathrm{QG}, 2}>10^{11} \mathrm{GeV}$ [31]. Unfortunately, as it stands, this argument only works if the UV theory is supersymmetric, which requires Hořava gravity to be supersymmetric as well. It is not known whether such a supersymmetrization exists.

An alternative was suggested in Ref. [19], in which it was proposed that supersymmetry breaking in the matter sector could be initiated by LV gravity. As a result, both SUSY breaking and Lorentz violation arise from the same coupling, and the contributions of graviton loops are controlled by a single dimensionless ratio,

$$
\frac{\delta c}{c} \sim \frac{\Lambda_{\mathrm{HL}}^{2}}{M_{p}^{2}}, \quad m_{\mathrm{soft}}^{2} \sim \Lambda_{\mathrm{UV}}^{2} \cdot \frac{\Lambda_{\mathrm{HL}}^{2}}{M_{p}^{2}} .
$$

The analysis of Ref. [19] does not, however, take into account the loop contribution from the vector modes of the gravity sector. As these were the source of difficulty in our analysis and in the analysis of Ref. [14], an alternative mechanism-such as the mixed derivative terms discussed in Sec. IVA-is presumably required to guarantee naturalness.

We comment briefly on an additional possibility. The power-law divergence of $\delta c^{2}$ discussed in Sec. III E can be attributed, in part, not to a non-Lifshitz dispersion relation of $N_{i}$, but rather to the scaling behavior of the matter field. The divergence can, however, be reduced by increasing the dimension of the inverse scalar propagator, corresponding to the introduction of higher-dimension LV operators. The authors of Ref. [14] avoided introducing higher-dimension LV operators because in typical field theories loop effects lead to $O(1)$ values of $\delta c^{2}$. Since SUSY protects the infrared value of $\delta c^{2}$, however, if the split in scales between SUSY breaking and Lorentz violation is sufficiently large, it may be possible to suppress LV effects from matter loops and gravity loops simultaneously. If SUSY is broken as in Ref. [19] by interaction with Hořava gravity, it is possible that naturalness can be preserved without mixed derivative terms. We leave a detailed investigation of this scenario to future work.

\section{Strong dynamics}

Another proposal is that if one allows for strong dynamics then Lorentz invariance can be restored in the infrared $[10,12,13]$. Suppose we have a matter sector with modes which have different sound speeds and are coupled with coupling constant $g$. Parametrizing their relative speed difference as

$$
\eta=\frac{\beta_{1}}{\beta_{2}}-1,
$$

near $\beta_{1} \approx \beta_{2} \approx c$, one has the typical behavior

$$
\frac{\partial \eta}{\partial \log \mu}=\frac{b}{4 \pi^{2} c^{3}} g^{2} \eta
$$

where $\mu$ is the renormalization scale and $b=O(1)$ [12]. If $g$ is a classically marginal coupling, then it will run logarithmically, $g^{2} \sim(A+B \log M / \mu)^{-1}$, causing $\eta$ to run according to

$$
\eta \sim \frac{1}{(A+B \log M / \mu)^{\#}},
$$

where $M$ is some large mass scale [12]. In this case, $\eta$ may flow to zero, but much too slowly to be consistent with observational constraints. If the coupling constant does not run, however, then $\eta$ runs with a power law of which the exponent is proportional to $g^{2}$, as

$$
\eta \propto\left(\frac{\mu}{M}\right)^{\frac{b}{4 \pi^{2} c^{2}} g^{2}} .
$$

Thus, when two modes spend a long RG time near a strongly coupled fixed point, their sound speeds rapidly converge as we flow to the infrared $[12,13]^{7}$

Finally, if a similar mechanism functions for the gravity sector as well, it might help fulfill the phenomenological constraints on the renormalization group flow presented in (54) and (55).

Before concluding, it is worth commenting on the possibility of reducing the degree of divergence by introducing additional fields unrelated to any symmetry [unlike the $U(1)$ extension or a hypothetical supersymmetric version of Horava gravity]. As it is the vector modes which are problematic, the symmetry of the background requires we introduce a vector field $B_{i}$. To modify the propagators, we should couple $B_{i}$ to $N_{i}$ locally (the theory should be local before gauge fixing), but such a coupling is strongly constrained by the foliation preserving diffeomorphisms and CPT symmetry. Given the existence of a suitable coupling, there is another problem. To suppress the problematic divergence requires the diagonalized vector system to have propagators that scale like $1 / k^{2 z}$ (or be suppressed even further) in the UV. This then requires the new spin-1 mode to have a Lifshitz scaling propagator with

\footnotetext{
${ }^{7}$ Another construction that speeds up the flow is to introduce many additional matter/gauge fields [12]. This can lead effectively to a power-law running, but it requires the addition of a large hidden sector, together with a mechanism decoupling it at experimental energies from the Standard Model fields. It is therefore unlikely that this possibility is feasible phenomenologically.
} 
$z \geq D$. Without some symmetry principle, one could then write infinitely many independent terms, and thus the theory would be nonpredictive, and hence we do not find this a viable option.

\section{SUMMARY}

This paper considered the mechanism proposed in Ref. [14] for obtaining a matter sector with natural IR Lorentz invariance coupled to nonprojectable Hořava gravity and applied it to the projectable theory. We showed that, as in the nonprojectable model, in the absence of additional ingredients, this mechanism is spoiled by contributions from the vector modes. Our analysis, valid for any number of spatial dimensions $D$, shows that the problem exists for any $D>2$ and grows worse as $D$ increases. ${ }^{8}$ In particular, the naturalness problem cannot be avoided by compactifying a higher-dimensional theory. Our computation furthermore used the gauge fixing of Ref. [18], in which all propagators are regular and have Lifshitz scaling, further supporting the results of Ref. [14] and suggesting that something more than the irregularity of the shift propagator lies at the heart of the mechanism's failure. Namely, the source of difficulty is simply the lack of Lifshitz scaling in the matter sector in the UV, combined with the fact that the shift propagator in the UV is less suppressed than the graviton propagator.

We discussed several possible approaches to this problem. One modification proposed already in Ref. [14] was the addition of mixed derivative terms, which for the nonprojectable theory was found in Ref. [17] to generate a new propagating d.o.f. with IR instabilities. As a result, the success of the mechanism relies on whether the IR couplings satisfy the stability condition (55). The offending extra mode does not exist in the projectable model with mixed derivatives, but the gradient (IR) instability of the original model without mixed derivatives persists. For this model to have viable phenomenology requires both an analogue of the Vainshtein mechanism and the couplings to flow in the IR to values satisfying the phenomenological constraints (55) [5]. We leave the investigation of these issues to future work.

A second possibility, proposed in Refs. [11,14,19], is based on supersymmetry. It is therefore interesting to seek a supersymmetric version of Hořava gravity. (For work relevant to this direction, see Refs. [32,33] on the supersymmetrization of field theories with Lifshitz scaling and the æther vector, respectively.) Meanwhile, one conceivable solution would combine UV supersymmetry in the matter sector (without Lorentz invariance), together with soft SUSY breaking by interaction with Hořava gravity.

\footnotetext{
${ }^{8}$ Curiously, although power counting suggests a linear dependence on $\Lambda$ for $D=2$, these terms cancel, and we find only logarithmic divergences in this case. We have only verified this special feature for spins 0 and 1 .
}

The remaining mechanism appearing in the literature posits that RG flow is responsible for IR emergent Lorentz symmetry $[10,12,13]$. For weakly coupled models, the flow is too slow to be phenomenologically viable, requiring the RG flow to pass near a strongly coupled fixed point. Such behavior is expected in some beyond-Standard Model scenarios, such as walking technicolor. Detailed models with the necessary properties thus need to be developed and studied.

Let us now comment on the scenario of emergent Lorentz symmetry and its relation to our results. In the literature [10,12,13], emergent Lorentz symmetry was investigated without including higher spatial derivative terms. We thus need to extend the analysis to systems with higher spatial derivative terms, so that all fields enjoy the Lifshitz scaling with a common value of the dynamical critical exponent $z$ in the UV. That is, we need to consider dispersion relations satisfying $\omega^{2} \simeq k^{2 z} / M^{2(z-1)}$ for $k \gg M$ and $\omega^{2} \simeq c_{s}^{2} k^{2}$ for $k \ll M$, where $M$ is the suppression scale of the higher spatial derivative term. In principle, $M$ can be different for different species. The main result of the present paper, namely the failure of the mechanism proposed in Ref. [14], clearly excludes the case with $M_{\text {matter }} \gg M_{\text {grav }}$ since in this case the matter sector would have the $z=1$ scaling all the way up to $\Lambda \sim M_{\text {matter }}$ as in the mechanism of Ref. [14]. On the other hand, if $M_{\text {matter }} \sim M_{\text {grav }}$, then the main result of the present paper does not apply to the scenario of emergent Lorentz symmetry, and it is generically expected that Lorentz invariance in the matter sector may emerge at low energy as a consequence of the RG flow. In summary, the results of the present paper suggest that not only the gravity sector but also the matter sector should exhibit Lifshitz scaling above some scale and that there should not be a large separation between the transition scales in the gravity and matter sectors.

Our discussion so far has been limited to the matter sector. The recent multimessenger observations of a binary neutron star merger [34], however, constrain the difference between the speed of gravitational waves $c_{\mathrm{gw}}$ and the speed of light $c_{\gamma}$ to the level of $O\left(10^{-15}\right)$ [6,35-39]. In the context of the scenario of emergent Lorentz symmetry, it is therefore important to see under what conditions (if any) there can be a fast RG flow toward $c_{\mathrm{gw}}=c_{\gamma}$.

It is, finally, worth comparing the naturalness of Lorentz symmetry to some better-known naturalness problems in cosmology: the cosmological constant and curvature problems. The cosmological constant is relevant in the IR, so the cosmological constant problem should be more severe than the curvature problem (as evidenced by the ability of inflation to solve the latter and not the former). Since $\lambda$ and $c_{\mathrm{gw}}$ are classically marginal in the IR, power counting suggests that the naturalness problems discussed in this paper will also be less severe than the cosmological constant problem. 


\section{ACKNOWLEDGMENTS}

The authors would like to thank M. Pospelov for helpful correspondence. S. M. thanks the L'Institut Denis Poisson (LMPT) for hospitality. A. C. is grateful for the hospitality of the Yukawa Institute of Theoretical Physics where this work started and also to the University of Nottingham. The work of A.C. was undertaken in part as an overseas researcher under a Short-Term Fellowship of the Japan Society for the Promotion of Sciences. The work of C. M. T. was supported by the Thousand Young Talents Program, Fudan University, and a Humboldt Research Fellowship from the Alexander von Humboldt Foundation. The work of S. M. was supported by Japan Society for the Promotion of Science (JSPS) Grants-in-Aid for Scientific Research (KAKENHI) No. 17H02890 and No. $17 \mathrm{H} 06359$ and by World Premier International Research Center Initiative (WPI), MEXT, Japan.

\section{APPENDIX: REGULATOR AND LOOP INTEGRALS}

We regulate the integrals introduced in Sec. II D as follows. We first integrate over all $\omega=P_{0}$ and then cut off the upper limit of the $p$ integrals at $p=\Lambda$, where $p=\sqrt{\delta^{i j} p_{i} p_{j}}$ and $p_{i}=P_{i}$. While this regularization scheme is not gauge invariant and modifies the long-range behavior of propagators, it suffices for the purpose of computing the general behavior of ultraviolet divergences, as we are taking $\Lambda^{D} \gg \Lambda M_{*}^{D-1}$, where $M_{*}$ is the scale at which anisotropic scaling begins to dominate. With this regularization scheme, we have

$$
\begin{aligned}
a^{-1} \mathcal{J}_{1} & =\int \frac{d \omega d^{D} p}{(2 \pi)^{D+1}} \frac{p^{2(D-1)}}{\omega^{2}+\alpha^{2} p^{2 D}}=\int \frac{d^{D} p}{2^{D+1} \pi^{D}} \frac{p^{D-2}}{\alpha} \\
& =\frac{\Lambda^{2(D-1)}}{2^{D+1} \pi^{D / 2} \Gamma(D / 2)(D-1) \alpha}, \\
a^{-1} \mathcal{J}_{2} & =\int \frac{d \omega d^{D} p}{(2 \pi)^{D+1}} \frac{\omega^{2} p^{2(D-1)}}{\left(\omega^{2}+\beta^{2} p^{2}\right)\left(\omega^{2}+\alpha^{2} p^{2 D}\right)} \\
& =\int \frac{d^{D} p}{2^{D+1} \pi^{D}} \frac{p^{2(D-1)}}{\alpha p^{D}+\beta k} \\
& =\frac{\left(\frac{\Lambda^{2(D-1)}}{2}-\Lambda^{(D-1)} \frac{\beta}{\alpha}+\frac{\beta^{2}}{\alpha^{2}} \log \frac{\alpha}{\beta} \Lambda^{D-1}\right)}{2^{D} \pi^{D / 2} \Gamma(D / 2)(D-1) \alpha}, \\
a^{-1} \mathcal{J}_{3} & =\int \frac{d \omega d^{D} p}{(2 \pi)^{D+1}} \frac{p^{2 D}}{\left(\omega^{2}+\beta^{2} p^{2}\right)\left(\omega^{2}+\alpha^{2} p^{2 D}\right)} \\
& =\int \frac{d^{D} p}{2^{D+1} \pi^{D}} \frac{p^{D-1}}{\alpha \beta\left(\alpha p^{D}+k \beta\right)} \\
& =\frac{\left(\frac{\Lambda^{D-1}}{\beta}-\frac{1}{\alpha} \log \left(\frac{\alpha}{\beta} \Lambda^{D-1}\right)\right)}{2^{D} \pi^{D / 2} \Gamma(D / 2)(D-1) \alpha^{2}},
\end{aligned}
$$

having dropped any convergent pieces, where $\mathcal{J}_{1,2,3}, a$, and $\alpha$ stand for $\mathcal{J}_{1,2,3}^{(I)}, a_{I}$, and $\alpha_{I}(>0)(I=1,2)$, respectively,

$$
\begin{aligned}
a^{\prime-1} \mathcal{J}_{4}= & \int \frac{d \omega d^{D} p}{(2 \pi)^{D+1}} \frac{1}{\omega^{2}+\alpha^{\prime 2} p^{2 D}}=\int \frac{d^{D} p}{2^{D+1} \pi^{D}} \frac{1}{\alpha^{\prime} p^{D}} \\
= & \frac{1}{2^{D} \pi^{D / 2} \Gamma(D / 2) \alpha^{\prime}} \log \left(\frac{\Lambda}{m}\right) \\
a^{\prime-1} \mathcal{J}_{5} & =\int \frac{d \omega d^{D} p}{(2 \pi)^{D+1}} \frac{1}{\omega^{2}+\alpha^{\prime 2} p^{2 D}} \frac{\omega^{2}}{\omega^{2}+\beta^{2} p^{2}} \\
& =\int \frac{d^{D} p}{2^{D+1} \pi^{D}} \frac{1}{\alpha^{\prime} p^{D}+\beta k} \\
& =\frac{\log \left(\frac{\alpha^{\prime}}{\beta} \Lambda^{D-1}\right)}{2^{D} \pi^{D / 2} \Gamma(D / 2)(D-1) \alpha^{\prime}}, \\
a^{\prime-1} \mathcal{J}_{6} & =\int \frac{d \omega d^{D} p}{(2 \pi)^{D+1}} \frac{1}{\omega^{2}+\alpha^{\prime 2} p^{2 D}} \frac{p^{2}}{\omega^{2}+\beta^{2} p^{2}} \\
& =\int \frac{d^{D} p}{2^{D+1} \pi^{D}} \frac{1}{\alpha^{\prime} \beta p^{D-1}} \frac{1}{\alpha^{\prime} p^{D}+\beta k} \\
& =(\text { finite in } \Lambda),
\end{aligned}
$$

again having dropped any convergent pieces and where $\mathcal{J}_{4,5,6}, a^{\prime}$, and $\alpha^{\prime}$ stand for $\mathcal{J}_{4,5,6}^{(n)}, a_{n}^{\prime}$, and $\alpha_{n}^{\prime}(>0)$, respectively, and

$$
\begin{aligned}
\mathcal{J}_{7} & =\int \frac{d \omega d^{D} p}{(2 \pi)^{D+1}} \frac{1}{\omega^{2}+\beta^{2} p^{2}} \\
& =\frac{\Lambda^{D-1}}{2^{D} \pi^{D / 2} \Gamma(D / 2)(D-1) \beta} .
\end{aligned}
$$

The integrals $\mathcal{J}_{4}$ and $\mathcal{J}_{6}$ suffer from infrared divergences, so we have introduced an IR cutoff $m$. As integral $\mathcal{J}_{6}$ has only infrared divergences, its inclusion is not required for an analysis of UV properties, but presumably it is required if one wants to keep track of the cancellation of infrared divergences.

We also have the following identities,

$$
\mathcal{J}_{2}+\beta^{2} \mathcal{J}_{3}=\mathcal{J}_{1}, \quad \mathcal{J}_{5}+\beta^{2} \mathcal{J}_{6}=\mathcal{J}_{4}
$$

and the following approximate relation that holds up to parts which vanish as $\Lambda \rightarrow \infty$ and $m \rightarrow 0$ :

$$
\frac{\mathcal{J}_{5}}{a^{\prime}}+\alpha^{2} \frac{\mathcal{J}_{3}}{a}=\mathcal{J}_{7}+\frac{\left(\alpha-\alpha^{\prime}\right)\left[\ln \left(\frac{\alpha}{\beta} \Lambda^{D-1}\right)\right]}{2^{D} \pi^{D / 2} \Gamma(D / 2)(D-1) \alpha \alpha^{\prime}} .
$$


[1] P. Hořava, Quantum gravity at a Lifshitz point, Phys. Rev. D 79, 084008 (2009).

[2] M. Visser, Lorentz symmetry breaking as a quantum field theory regulator, Phys. Rev. D 80, 025011 (2009).

[3] S. Mukohyama, Scale-invariant cosmological perturbations from Hořava-Lifshitz gravity without inflation, J. Cosmol. Astropart. Phys. 06 (2009) 001.

[4] S. F. Bramberger, A. Coates, J. Magueijo, S. Mukohyama, R. Namba, and Y. Watanabe, Solving the flatness problem with an anisotropic instanton in Hořava-Lifshitz gravity, Phys. Rev. D 97, 043512 (2018).

[5] S. Mukohyama, Hořava-Lifshitz cosmology: A review, Classical Quantum Gravity 27, 223101 (2010).

[6] A. E. Gümrükçüoğlu, M. Saravani, and T. P. Sotiriou, Hořava gravity after GW170817, Phys. Rev. D 97, 024032 (2018).

[7] O. Ramos and E. Barausse, Constraints on Hořava gravity from binary black hole observations, Phys. Rev. D 99, 024034 (2019).

[8] I. Kharuk and S. Sibiryakov, Emergent Lorentz invariance with chiral fermions, Theor. Math. Phys. 189, 1755 (2016).

[9] N. Afshordi, Why is high energy physics Lorentz invariant, arXiv: 1511.07879.

[10] S. Chadha and H. B. Nielsen, Lorentz invariance as a lowenergy phenomenon, Nucl. Phys. B217, 125 (1983).

[11] S. G. Nibbelink and M. Pospelov, Lorentz Violation in Supersymmetric Field Theories, Phys. Rev. Lett. 94, 081601 (2005).

[12] M. M. Anber and J.F. Donoghue, The emergence of a universal limiting speed, Phys. Rev. D 83, 105027 (2011).

[13] G. Bednik, O. Pujols, and S. Sibiryakov, Emergent Lorentz invariance from strong dynamics: Holographic examples, J. High Energy Phys. 11 (2013) 064.

[14] M. Pospelov and Y. Shang, Lorentz violation in Hořava-Lifshitz-type theories, Phys. Rev. D 85, 105001 (2012).

[15] M. Colombo, A. E. Gümrükçüoğlu, and T. P. Sotiriou, Hořava gravity with mixed derivative terms: Power counting renormalizability with lower order dispersions, Phys. Rev. D 92, 064037 (2015).

[16] M. Colombo, A. E. Gümrükçüoğlu, and T. P. Sotiriou, Hořava gravity with mixed derivative terms, Phys. Rev. D 91, 044021 (2015).

[17] A. Coates, M. Colombo, A. E. Gümrükçüoğlu, and T. P. Sotiriou, Uninvited guest in mixed derivative Hořava gravity, Phys. Rev. D 94, 084014 (2016).

[18] A. O. Barvinsky, D. Blas, M. Herrero-Valea, S. M. Sibiryakov, and C. F. Steinwachs, Renormalization of Hořava gravity, Phys. Rev. D 93, 064022 (2016).

[19] M. Pospelov and C. Tamarit, Lifshitz-sector mediated SUSY breaking, J. High Energy Phys. 01 (2014) 048.

[20] K. Izumi and S. Mukohyama, Nonlinear superhorizon perturbations in Hořava-Lifshitz gravity, Phys. Rev. D 84, 064025 (2011).

[21] A. E. Gümrükçüoğlu, S. Mukohyama, and A. Wang, General relativity limit of Hořava-Lifshitz gravity with a scalar field in gradient expansion, Phys. Rev. D 85, 064042 (2012).
[22] S. Mukohyama, Dark matter as integration constant in Hořava-Lifshitz gravity, Phys. Rev. D 80, 064005 (2009).

[23] S. Mukohyama, Caustic avoidance in HořavaLifshitz gravity, J. Cosmol. Astropart. Phys. 09 (2009) 005 .

[24] D. Blas, O. Pujolas, and S. Sibiryakov, On the extra mode and inconsistency of Hořava gravity, J. High Energy Phys. 10 (2009) 029.

[25] P. Hořava and C. M. Melby-Thompson, General covariance in quantum gravity at a Lifshitz point, Phys. Rev. D 82, 064027 (2010).

[26] K. Lin, S. Mukohyama, A. Wang, and T. Zhu, PostNewtonian approximations in the Hořava-Lifshitz gravity with extra U(1) symmetry, Phys. Rev. D 89, 084022 (2014).

[27] S. Mukohyama, R. Namba, R. Saitou, and Y. Watanabe, Hamiltonian analysis of nonprojectable Hořava-Lifshitz gravity with $U(1)$ symmetry, Phys. Rev. D 92, 024005 (2015).

[28] P. A. Bolokhov, S. Groot Nibbelink, and M. Pospelov, Lorentz violating supersymmetric quantum electrodynamics, Phys. Rev. D 72, 015013 (2005).

[29] K. Toma et al., Strict Limit on CPT Violation from Polarization of Gamma-Ray Burst, Phys. Rev. Lett. 109, 241104 (2012).

[30] G. Amelino-Camelia, J. R. Ellis, N. E. Mavromatos, D. V. Nanopoulos, and S. Sarkar, Tests of quantum gravity from observations of gamma-ray bursts, Nature (London) 393, 763 (1998).

[31] M. Ackermann et al. (Fermi GBM/LAT Collaboration), A limit on the variation of the speed of light arising from quantum gravity effects, Nature (London) 462, 331 (2009).

[32] D. Redigolo, Lorentz-violating supersymmetric quantum field theories, Phys. Rev. D 85, 085009 (2012).

[33] O. Pujolas and S. Sibiryakov, Supersymmetric aether, J. High Energy Phys. 01 (2012) 062.

[34] B. P. Abbott et al. (SKA South Africa/MeerKAT, Virgo, Fermi GBM, INTEGRAL, IceCube, IPN, Insight-Hxmt, ANTARES, Swift and Dark Energy Camera GW-EM, Dark Energy Survey, DLT40, GRAWITA, Fermi-LAT, ATCA, ASKAP, OzGrav, DWF (Deeper Wider Faster Program), AST3, CAASTRO, VINROUGE, MASTER, J-GEM, GROWTH, JAGWAR, CaltechNRAO, TTU-NRAO, NuSTAR, Pan-STARRS, KU, Nordic Optical Telescope, ePESSTO, GROND, Texas Tech University, TOROS, BOOTES, MWA, CALET, IKI-GW Follow-up, H.E.S.S., LOFAR, LWA, HAWC, Pierre Auger, ALMA, Pi of Sky, DFN, ATLAS, High Time Resolution Universe Survey, RIMAS, RATIR, SKA South Africa/MeerKAT Collaborations, AstroSat Cadmium Zinc Telluride Imager Team, AGILE Team, 1M2H, and Las Cumbres Observatory Group, MAXI Team, TZAC Consortium, SALT Group, Euro VLBI Team, and Chandra Team at McGill University Collaborations), Multi-messenger observations of a binary neutron star merger, Astrophys. J. 848, L12 (2017).

[35] P. Creminelli and F. Vernizzi, Dark energy after GW170817, Phys. Rev. Lett. 119, 251302 (2017). 
[36] J. M. Ezquiaga and M. Zumalacárregui, Dark Energy After GW170817: Dead Ends and the Road Ahead, Phys. Rev. Lett. 119, 251304 (2017).

[37] T. Baker, E. Bellini, P. G. Ferreira, M. Lagos, J. Noller, and I. Sawicki, Strong constraints on cosmological gravity from GW170817 and GRB 170817A, Phys. Rev. Lett. 119, 251301 (2017).
[38] J. Sakstein and B. Jain, Implications of the Neutron Star Merger GW170817 for Cosmological Scalar-Tensor Theories, Phys. Rev. Lett. 119, 251303 (2017).

[39] J. Oost, S. Mukohyama, and A. Wang, Constraints on Einstein-aether theory after GW170817, Phys. Rev. D 97, 124023 (2018). 